\title{
Monotone Iterative Technique for the Initial Value Problems of Impulsive Evolution Equations in Ordered Banach Spaces
}

\author{
He Yang \\ Department of Mathematics, Northwest Normal University, Lanzhou 730070, China \\ Correspondence should be addressed to He Yang, yanghe256@163.com \\ Received 15 March 2010; Accepted 11 July 2010 \\ Academic Editor: Paul Eloe \\ Copyright (C) 2010 He Yang. This is an open access article distributed under the Creative Commons \\ Attribution License, which permits unrestricted use, distribution, and reproduction in any \\ medium, provided the original work is properly cited. \\ This paper deals with the existence and uniqueness of mild solutions for the initial value problems \\ of abstract impulsive evolution equations in an ordered Banach space $E: u^{\prime}(t)+A u(t)=$ \\ $f(t, u(t), G u(t)), t \in[0, a], t \neq t_{k},\left.\Delta u\right|_{t=t_{k}}=I_{k}\left(u\left(t_{k}\right)\right), 0<t_{1}<t_{2}<\cdots<t_{m}<a, u(0)=u_{0}$, where \\ $A: D(A) \subset E \rightarrow E$ is a closed linear operator, and $f:[0, a] \times E \times E \rightarrow E$ is a nonlinear mapping. \\ Under wide monotone conditions and measure of noncompactness conditions of nonlinearity $f$, \\ some existence and uniqueness results are obtained by using a monotone iterative technique in the \\ presence of lower and upper solutions.
}

\section{Introduction and Main Results}

Differential equations involving impulse effects occur in many applications: physics, population dynamics, ecology, biological systems, biotechnology, industrial robotic, pharmacokinetics, optimal control, and so forth. Therefore, it has been an object of intensive investigation in recent years; see, for instance, the monographs [1-5]. Correspondingly, the existence of mild solutions of impulsive evolution differential equations has also been studied by several authors; see [6-8]. However, the theory still remains to be developed.

In this paper, we use a monotone iterative technique in the presence of lower and upper solutions to discuss the existence of mild solutions for the initial value problem (IVP) of first-order nonlinear impulsive evolution equations

$$
\begin{gathered}
u^{\prime}(t)+A u(t)=f(t, u(t), G u(t)), \quad t \in J, t \neq t_{k}, \\
\left.\Delta u\right|_{t=t_{k}}=I_{k}\left(u\left(t_{k}\right)\right), \quad k=1,2, \ldots, m, \\
u(0)=u_{0}
\end{gathered}
$$


in an ordered Banach space $E$, where $A: D(A) \subset E \rightarrow E$ is a closed linear operator, $-A$ generates a $C_{0}$-semigroup $T(t)(t \geq 0)$ in $E, f: J \times E \times E \rightarrow E$ is a nonlinear mapping, $J=[0, a], a>0$ is a constant, $0=t_{0}<t_{1}<t_{2}<\cdots<t_{m}<t_{m+1}=a, I_{k}: E \rightarrow E$ is an impulsive function, $k=1,2, \ldots, m, u_{0} \in E$, and

$$
G u(t)=\int_{0}^{t} K(t, s) u(s) d s
$$

is a Volterra integral operator with integral kernel $K \in C\left(\Delta, \mathbb{R}^{+}\right), \quad \Delta=\{(t, s) \mid 0 \leq s \leq t \leq a\}$, $\left.\Delta u\right|_{t=t_{k}}$ denotes the jump of $u(t)$ at $t=t_{k}$, that is, $\left.\Delta u\right|_{t=t_{k}}=u\left(t_{k}^{+}\right)-u\left(t_{k}^{-}\right)$, where $u\left(t_{k}^{+}\right)$and $u\left(t_{k}^{-}\right)$ represent the right and left limits of $u(t)$ at $t=t_{k}$, respectively. Let PC $(J, E)=\{u: J \rightarrow E \mid u(t)$ is continuous at $t \neq t_{k}$, and left continuous at $t=t_{k}$, and $u\left(t_{k}^{+}\right)$exists, $\left.k=1,2, \ldots, m\right\}$. Evidently, $\mathrm{PC}(J, E)$ is a Banach space with norm $\|u\|_{\mathrm{PC}}=\sup _{t \in J}\|u(t)\|$. Let $J^{\prime}=J \backslash\left\{t_{1}, t_{2}, \ldots, t_{m}\right\}$. An abstract function $u \in \mathrm{PC}(J, E) \cap C^{1}\left(J^{\prime}, E\right) \cap C\left(J^{\prime}, E_{1}\right)\left(E_{1}\right.$ is a Banach space with norm $\|x\|_{1}=$ $\|x\|+\|A x\|)$ is called a solution of $\operatorname{IVP}(1.1)$, if $u(t)$ satisfies all the equalities of (1.1).

Let $E$ be an ordered Banach space with norm $\|\cdot\|$ and partial order $\leq$, whose positive cone $P=\{x \in E \mid x \geq 0\}$ is normal with a normal constant $N$. If an abstract function $v \in \mathrm{PC}(J, E) \cap C^{1}\left(J^{\prime}, E\right) \cap C\left(J^{\prime}, E_{1}\right)$ satisfies

$$
\begin{gathered}
v^{\prime}(t)+A v(t) \leq f(t, v(t), G v(t)), \quad t \in J, \quad t \neq t_{k} \\
\left.\Delta v\right|_{t=t_{k}} \leq I_{k}\left(v\left(t_{k}\right)\right), \quad k=1,2, \ldots, m \\
v(0) \leq u_{0}
\end{gathered}
$$

we call it a lower solution of IVP(1.1). If all the inequalities of (1.3) are inverse, we call it an upper solution of IVP(1.1).

In 1999, Liu [6], by means of the semigroup theory, has proved the existence and uniqueness of mild solutions for $\operatorname{IVP}(1.1)$ when $f=f(t, u)$. He demands that the nonlinear term $f$ and the impulsive function $I_{k}$ satisfy the following conditions:

$$
\begin{gathered}
\|f(t, u)-f(t, v)\| \leq C\|u-v\|, \quad t \in J, u, v \in E, \\
\left\|I_{k}(u)-I_{k}(v)\right\| \leq h_{k}\|u-v\|, \quad u, v \in E,
\end{gathered}
$$

where $C$ and $h_{k}(k=1,2, \ldots, m)$ are positive constants and satisfy

$$
M^{*}\left(\mathrm{C} a+\sum_{k=1}^{m} h_{k}\right)<1,
$$

where $M^{*}=\max _{t \in J}\|T(t)\|$. Inequality (1.6) is a strongly restricted condition, and it is difficult to satisfy in applications.

Recently, Anguraj and Arjunan [7], under similar assumptions of [6], have obtained a unique mild solution for $\operatorname{IVP}(1.1)$ when $f=f(t, u, G u, S u)$. Cardinali and Rubbioni [8] have discussed the existence of mild solutions for the impulsive evolution differential inclusions under the measure of noncompactness conditions on every bounded set $D \subset E$. However the assumptions in these papers are also difficult to satisfy in applications. 
The purpose of this paper is to improve and extend the above mentioned results. We will delete the Lipschitz condition (1.5) for impulsive function $I_{k}$ and the restriction condition (1.6) and improve condition (1.4) for nonlinear term $f$. Our main results are as follows.

Theorem 1.1. Let $E$ be an ordered Banach space, whose positive cone $P$ is normal, $A: D(A) \subset E \rightarrow$ $E$ be a closed linear operator, $-A$ generate a positive $C_{0}$-semigroup $T(t)(t \geq 0), f \in C(J \times E \times E, E)$, and $I_{k} \in C(E, E), k=1,2, \ldots, m$. If IVP(1.1) has a lower solution $v_{0}$ and an upper solution $w_{0}$ with $v_{0} \leq w_{0}$ and the following conditions are satisfied:

$\left(H_{1}\right)$ there exists a positive constant $C$ such that

$$
f\left(t, x_{2}, y_{2}\right)-f\left(t, x_{1}, y_{1}\right) \geq-C\left(x_{2}-x_{1}\right),
$$

for any $t \in J, v_{0}(t) \leq x_{1} \leq x_{2} \leq w_{0}(t)$, and $G v_{0}(t) \leq y_{1} \leq y_{2} \leq G w_{0}(t)$,

$\left(H_{2}\right)$ for any $x_{1}, x_{2} \in E$ with $v_{0}\left(t_{k}\right) \leq x_{1} \leq x_{2} \leq w_{0}\left(t_{k}\right), k=1,2, \ldots, m$, one has

$$
I_{k}\left(x_{1}\right) \leq I_{k}\left(x_{2}\right),
$$

$\left(H_{3}\right)$ there exists a positive constant $L$ such that

$$
\alpha\left(\left\{f\left(t, x_{n}, y_{n}\right)\right\}\right) \leq L\left(\alpha\left(\left\{x_{n}\right\}\right)+\alpha\left(\left\{y_{n}\right\}\right)\right),
$$

for any $t \in J$, and increasing or decreasing monotonic sequences $\left\{x_{n}\right\} \subset\left[v_{0}(t), w_{0}(t)\right]$ and $\left\{y_{n}\right\} \in\left[G v_{0}(t), G w_{0}(t)\right]$.

Then IVP(1.1) has minimal and maximal mild solutions between $v_{0}$ and $w_{0}$, which can be obtained by a monotone iterative procedure starting from $v_{0}$ and $w_{0}$, respectively.

Clearly, condition $\left(\mathrm{H}_{3}\right)$ greatly improves the measure of noncompactness condition in [8]. Therefore, Theorem 1.1 greatly improves the main results in [6-8]. In Theorem 1.1, if Banach space $E$ is weakly sequentially complete, condition $\left(H_{3}\right)$ holds automatically; see [9, Theorem 2.2]. Hence, from Theorem 1.1, we have the following.

Corollary 1.2. Let $E$ be an ordered and weakly sequentially complete Banach space, whose positive cone $P$ is normal, $A: D(A) \subset E \rightarrow E$ be a closed linear operator, $-A$ generate a positive $C_{0}$-semigroup $T(t)(t \geq 0), f \in C(J \times E \times E, E)$, and $I_{k} \in C(E, E), k=1,2, \ldots, m$. If $\operatorname{IVP}(1.1)$ has a lower solution $v_{0}$ and an upper solution $w_{0}$ with $v_{0} \leq w_{0}$, and the conditions $\left(H_{1}\right)$ and $\left(H_{2}\right)$ are satisfied, then IVP $(1.1)$ has minimal and maximal mild solutions between $v_{0}$ and $w_{0}$, which can be obtained by a monotone iterative procedure starting from $v_{0}$ and $w_{0}$, respectively.

The proof of Theorem 1.1 will be shown in the next section. In Section 2, we also discuss the uniqueness of mild solutions for IVP(1.1) between the lower solution and upper solution (see Theorem 2.4). 


\section{Proof of the Main Results}

Let $C(J, E)$ denote the Banach space of all continuous $E$-value functions on interval $J$ with norm $\|u\|_{C}=\max _{t \in J}\|u(t)\|$ and let $C^{1}(J, E)$ denote the Banach space of all continuously differentiable $E$-value functions on interval $J$ with norm $\|u\|_{C^{1}}=\max \left\{\|u\|_{C^{\prime}},\left\|u^{\prime}\right\|_{C}\right\}$. Consider the initial value problem (IVP) of linear evolution equation without impulse

$$
\begin{gathered}
u^{\prime}(t)+A u(t)=h(t), \quad t \in J, \\
u(0)=u_{0} .
\end{gathered}
$$

It is well-known [10, chapter 4, Theorem 2.9], when $u_{0} \in D(A)$ and $h \in C^{1}(J, E), \operatorname{IVP}(2.1)$ has a classical solution $u \in C^{1}(J, E) \cap C\left(J, E_{1}\right)$ expressed by

$$
u(t)=T(t) u_{0}+\int_{0}^{t} T(t-s) h(s) d s, \quad t \in J
$$

Generally, when $u_{0} \in E$ and $h \in C(J, E)$, the function $u$ given by (2.2) belongs to $C(J, E)$ and it is called a mild solution of $\operatorname{IVP}(2.1)$.

Let us start by defining what we mean by a mild solution of problem

$$
\begin{gathered}
u^{\prime}(t)+A u(t)=h(t), \quad t \in J, \quad t \neq t_{k} \\
\left.\Delta u\right|_{t=t_{k}}=I_{k}\left(u\left(t_{k}\right)\right), \quad k=1,2, \ldots, m \\
u(0)=u_{0} .
\end{gathered}
$$

Definition 2.1. A function $u \in P C(J, E)$ is called a mild solution of $\operatorname{IVP}(2.3)$, if $u$ is a solution of integral equation

$$
u(t)=T(t) u_{0}+\int_{0}^{t} T(t-s) h(s) d s+\sum_{0<t_{k}<t} T\left(t-t_{k}\right) I_{k}\left(u\left(t_{k}\right)\right), \quad t \in J .
$$

To prove Theorem 1.1, for any $h \in \mathrm{PC}(J, E)$, we consider the linear initial value problem (LIVP) of impulsive evolution equation

$$
\begin{gathered}
u^{\prime}(t)+A u(t)+C u(t)=h(t), \quad t \in J, \quad t \neq t_{k}, \\
\left.\Delta u\right|_{t=t_{k}}=y_{k}, \quad k=1,2, \ldots, m, \\
u(0)=x,
\end{gathered}
$$

where $C \geq 0, x \in E$, and $y_{k} \in E, k=1,2, \ldots, m$. 
Lemma 2.2. For any $h \in P C(J, E), x \in E$, and $y_{k} \in E, k=1,2, \ldots, m, \operatorname{LIVP}(2.5)$ has a unique mild solution $u \in P C(J, E)$ given by

$$
u(t)=S(t) x+\int_{0}^{t} S(t-s) h(s) d s+\sum_{0<t_{k}<t} S\left(t-t_{k}\right) y_{k}, \quad t \in J
$$

where $S(t)=e^{-C t} T(t)(t \geq 0)$ is a $C_{0}$-semigroup generated by $-(A+C I)$.

Proof. Let $y_{0}=0$. If $u \in \mathrm{PC}(J, E)$ is a mild solution of $\operatorname{LIVP}(2.5)$, then the restriction of $u$ on $\left(t_{k-1}, t_{k}\right]$ satisfies the initial value problem of linear evolution equation without impulse

$$
\begin{gathered}
u^{\prime}(t)+A u(t)+C u(t)=h(t), \quad t_{k-1}<t \leq t_{k} \\
u\left(t_{k-1}^{+}\right)=u\left(t_{k-1}\right)+y_{k-1} .
\end{gathered}
$$

Hence, on $\left(t_{k-1}, t_{k}\right], u(t)$ can be expressed by

$$
u(t)=S\left(t-t_{k-1}\right) u\left(t_{k-1}\right)+S\left(t-t_{k-1}\right) y_{k-1}+\int_{t_{k-1}}^{t} S(t-s) h(s) d s
$$

Iterating successively in the above equality with $u\left(t_{j}\right)$ for $j=k-1, k-2, \ldots, 1,0$, we see that $u$ satisfies (2.6).

Inversely, we can verify directly that the function $u \in \mathrm{PC}(J, E)$ defined by (2.6) satisfies all the equalities of $\operatorname{LIVP}(2.5)$.

Let $\alpha(\cdot)$ denote the Kuratowskii measure of noncompactness of the bounded set. For the details of the definition and properties of the measure of noncompactness, see [11]. For any $B \subset C(J, E)$ and $t \in J$, set $B(t)=\{u(t) \mid u \in B\} \subset E$. If $B$ is bounded in $C(J, E)$, then $B(t)$ is bounded in $E$, and $\alpha(B(t)) \leq \alpha(B)$. In the proof of Theorem 1.1 we need the following lemma.

Lemma 2.3. Let $B=\left\{u_{n}\right\} \subset P C(J, E)$ be a bounded and countable set. Then $\alpha(B(t))$ is the Lebesgue integrable on $J$, and

$$
\alpha\left(\left\{\int_{J} u_{n}(t) d t\right\}\right) \leq 2 \int_{J} \alpha(B(t)) d t
$$

This lemma can be found in [12].

Evidently, $\mathrm{PC}(J, E)$ is also an ordered Banach space with the partial order " $\leq$ " reduced by the positive function cone $K_{\mathrm{PC}}=\{u \in \mathrm{PC}(J, E) \mid u(t) \geq 0, t \in J\}$. $K_{\mathrm{PC}}$ is also normal with the same normal constant $N$. For $v, w \in \operatorname{PC}(J, E)$ with $v \leq w$, we use $[v, w]$ to denote the ordered interval $\{u \in \operatorname{PC}(J, E) \mid v \leq u \leq w\}$ in $\operatorname{PC}(J, E)$, and use $[v(t), w(t)]$ to denote the ordered interval $\{x \in E \mid v(t) \leq x \leq w(t)\}$ in $E$. 
Proof of Theorem 1.1. Let $D=\left[v_{0}, w_{0}\right]$. We define a mapping $Q: D \rightarrow \operatorname{PC}(J, E)$ by

$$
(Q u)(t)=S(t) u_{0}+\int_{0}^{t} S(t-s)(f(s, u(s), G u(s))+C u(s)) d s+\sum_{0<t_{k}<t} S\left(t-t_{k}\right) I_{k}\left(u\left(t_{k}\right)\right), \quad t \in J .
$$

Clearly, $Q: D \rightarrow \operatorname{PC}(J, E)$ is continuous. By Lemma 2.2, the mild solution of $\operatorname{IVP}(1.1)$ is equivalent to the fixed point of operator $Q$. By assumptions $\left(H_{1}\right)$ and $\left(H_{2}\right), Q$ is increasing in $D$ and maps any bounded set in $D$ into a bounded set.

We show that $v_{0} \leq Q v_{0}, Q w_{0} \leq w_{0}$. Let $h(t) \triangleq v_{0}^{\prime}(t)+A v_{0}(t)+C v_{0}(t)$. By the definition of the lower solution, we easily see that $h \in \mathrm{PC}(J, E)$ and $h(t) \leq f\left(t, v_{0}(t), G v_{0}(t)\right)+C v_{0}(t)$ for $t \in J^{\prime}$. Because $v_{0}(t)$ is a solution of $\operatorname{LIVP}(2.5)$ for $x=v_{0}(0)$ and $y_{k}=\left.\Delta v_{0}\right|_{t=t_{k}}, \quad k=1,2, \ldots, m$, by Lemma 2.2 and the positivity of operator $S(t)(t \geq 0)$, we have

$$
\begin{aligned}
v_{0}(t)= & S(t) v_{0}(0)+\int_{0}^{t} S(t-s) h(s) d s+\left.\sum_{0<t_{k}<t} S\left(t-t_{k}\right) \Delta v_{0}\right|_{t=t_{k}} \\
\leq & S(t) u_{0}+\int_{0}^{t} S(t-s) h(s) d s+\sum_{0<t_{k}<t} S\left(t-t_{k}\right) I_{k}\left(v_{0}\left(t_{k}\right)\right) \\
= & S(t) u_{0}+\sum_{j=1}^{k} \int_{t_{j-1}}^{t_{j}} S(t-s) h(s) d s+\int_{t_{k}}^{t} S(t-s) h(s) d s+\sum_{0<t_{k}<t} S\left(t-t_{k}\right) I_{k}\left(v_{0}\left(t_{k}\right)\right) \\
\leq & S(t) u_{0}+\sum_{j=1}^{k} \int_{t_{j-1}}^{t_{j}} S(t-s)\left(f\left(s, v_{0}(s), G v_{0}(s)\right)+C v_{0}(s)\right) d s \\
& +\int_{t_{k}}^{t} S(t-s)\left(f\left(s, v_{0}(s), G v_{0}(s)\right)+C v_{0}(s)\right) d s+\sum_{0<t_{k}<t} S\left(t-t_{k}\right) I_{k}\left(v_{0}\left(t_{k}\right)\right) \\
= & S(t) u_{0}+\int_{0}^{t} S(t-s)\left(f\left(s, v_{0}(s), G v_{0}(s)\right)+C v_{0}(s)\right) d s+\sum_{0<t_{k}<t} S\left(t-t_{k}\right) I_{k}\left(v_{0}\left(t_{k}\right)\right) \\
= & \left(Q v_{0}\right)(t), \quad t \in J,
\end{aligned}
$$

namely, $v_{0} \leq Q v_{0}$. Similarly, it can be shown that $Q w_{0} \leq w_{0}$. Combining these facts with the increasing property of $Q$ in $D$, we see that $Q$ maps $D$ into itself, and $Q: D \rightarrow D$ is a continuously increasing operator.

Now, we define two sequences $\left\{v_{n}\right\}$ and $\left\{w_{n}\right\}$ in $D$ by the iterative scheme

$$
v_{n}=Q v_{n-1}, \quad w_{n}=Q w_{n-1}, \quad n=1,2, \ldots
$$

Then from the monotonicity of $Q$, it follows that

$$
v_{0} \leq v_{1} \leq \cdots \leq v_{n} \leq \cdots \leq w_{n} \leq \cdots \leq w_{1} \leq w_{0} .
$$

Next, we will show that $\left\{v_{n}\right\}$ and $\left\{w_{n}\right\}$ are uniformly convergent on $J$. 
For convenience, we denote $M=\max _{t \in J}\|S(t)\|, K_{0}=\max _{(t, s) \in \Delta} K(t, s)$. Let $J_{1}=\left[0, t_{1}\right]$, $J_{k}=\left(t_{k-1}, t_{k}\right], k=2,3, \ldots, m+1$ and let $B=\left\{v_{n} \mid n \in \mathbb{N}\right\}$ and $B_{0}=\left\{v_{n-1} \mid n \in \mathbb{N}\right\}$. From $B_{0}=B \cup\left\{v_{0}\right\}$, it follows that $\alpha\left(B_{0}(t)\right)=\alpha(B(t))$ for $t \in J$. Let

$$
\varphi(t):=\alpha(B(t))=\alpha\left(B_{0}(t)\right), \quad t \in J
$$

By Lemma 2.3, $\varphi(t)$ is Lebesgue integrable on $J$. Going from $J_{1}$ to $J_{m+1}$ interval by interval we show that $\varphi(t) \equiv 0$ on $J$.

For $t \in J$, there exists a $J_{k}$ such that $t \in J_{k}$. By (1.2) and Lemma 2.3, we have that

$$
\begin{aligned}
\alpha\left(G\left(B_{0}\right)(t)\right)= & \alpha\left(\left\{\int_{0}^{t} K(t, s) v_{n-1}(s) d s \mid n \in \mathbb{N}\right\}\right) \\
\leq & \sum_{j=1}^{k-1} \alpha\left(\left\{\int_{t_{j-1}}^{t_{j}} K(t, s) v_{n-1}(s) d s \mid n \in \mathbb{N}\right\}\right) \\
& +\alpha\left(\left\{\int_{t_{k-1}}^{t} K(t, s) v_{n-1}(s) d s \mid n \in \mathbb{N}\right\}\right) \\
\leq & 2 K_{0}\left(\sum_{j=1}^{k-1} \int_{t_{j-1}}^{t_{j}} \alpha\left(B_{0}(s)\right) d s+\int_{t_{k-1}}^{t} \alpha\left(B_{0}(s)\right) d s\right)=2 K_{0} \int_{0}^{t} \varphi(s) d s
\end{aligned}
$$

and therefore,

$$
\int_{0}^{t} \alpha\left(G\left(B_{0}\right)(s)\right) d s \leq 2 a K_{0} \int_{0}^{t} \varphi(s) d s
$$

For $t \in J_{1}$, from (2.10), (2.16), Lemma 2.3 and assumption $\left(H_{3}\right)$, we have

$$
\begin{aligned}
\varphi(t) & =\alpha(B(t))=\alpha\left(Q\left(B_{0}(t)\right)\right) \\
& =\alpha\left(\left\{\int_{0}^{t} S(t-s)\left(f\left(s, v_{n-1}(s), G v_{n-1}(s)\right)+C v_{n-1}(s)\right) d s \mid n \in \mathbb{N}\right\}\right) \\
& \leq 2 \int_{0}^{t} \alpha\left(\left\{S(t-s)\left(f\left(s, v_{n-1}(s), G v_{n-1}(s)\right)+C v_{n-1}(s)\right) \mid n \in \mathbb{N}\right\}\right) d s \\
& \leq 2 M \int_{0}^{t}\left(L\left(\alpha\left(B_{0}(s)\right)+\alpha\left(G\left(B_{0}\right)(s)\right)\right)+C \alpha\left(B_{0}(s)\right)\right) d s \\
& =2 M\left(L+C+2 a K_{0} L\right) \int_{0}^{t} \varphi(s) d s .
\end{aligned}
$$

By this and the Gronwall-Bellman inequality, we obtain that $\varphi(t) \equiv 0$ on $J_{1}$. In particular, $\alpha\left(B_{0}\left(t_{1}\right)\right)=\varphi\left(t_{1}\right)=0$, this means that $B_{0}\left(t_{1}\right)$ is precompact in $E$. Combining this with the continuity of $I_{1}$, it follows that $I_{1}\left(B_{0}\left(t_{1}\right)\right)$ is precompact in $E$, and $\alpha\left(I_{1}\left(B_{0}\left(t_{1}\right)\right)\right)=0$. 
Now, for $t \in J_{2}$, by (2.10) and the above argument for $t \in J_{1}$, we have

$$
\begin{aligned}
\varphi(t)= & \alpha(B(t))=\alpha\left(Q\left(B_{0}\right)(t)\right) \\
\leq & \alpha\left(\left\{\int_{0}^{t} S(t-s)\left(f\left(s, v_{n-1}(s), G v_{n-1}(s)\right)+C v_{n-1}(s)\right) d s \mid n \in \mathbb{N}\right\}\right) \\
& +\alpha\left(\left\{S\left(t-t_{1}\right) I_{1}\left(v_{n-1}\left(t_{1}\right)\right) \mid n \in \mathbb{N}\right\}\right) \\
\leq & 2 M\left(L+C+2 a K_{0} L\right) \int_{0}^{t} \varphi(s) d s+M \alpha\left(I_{1}\left(B_{0}\left(t_{1}\right)\right)\right) \\
= & 2 M\left(L+C+2 a K_{0} L\right) \int_{t_{1}}^{t} \varphi(s) d s .
\end{aligned}
$$

Again by the Gronwall-Bellman inequality, we obtain that $\varphi(t) \equiv 0$ on $J_{2}$, from which we obtain that $\alpha\left(B_{0}\left(t_{2}\right)\right)=0$ and $\alpha\left(I_{2}\left(B_{0}\left(t_{2}\right)\right)\right)=0$.

Continuing such a process interval by interval up to $J_{m+1}$, we can prove that $\varphi(t) \equiv 0$ on every $J_{k}, k=1,2, \ldots, m+1$. This means that $\left\{v_{n}(t)\right\}$ is precompact in $E$ for every $t \in J_{k}$. Hence $\left\{v_{n}(t)\right\}$ has a convergent subsequence in $E$. Combining this fact with the monotonicity (2.13), we easily prove that $\left\{v_{n}(t)\right\}$ itself is convergent in $E$, that is, there exists $\underline{u}(t) \in E$ such that $v_{n}(t) \rightarrow \underline{u}(t)$ as $n \rightarrow \infty$ for every $t \in J_{k}$. On the other hand, for any $t \in J_{k}$, we have

$$
v_{n}(t)=S(t) u_{0}+\int_{0}^{t} S(t-s)\left(f\left(s, v_{n-1}(s), G v_{n-1}(s)\right)+C v_{n-1}(s)\right) d s+\sum_{0<t_{i}<t} S\left(t-t_{i}\right) I_{i}\left(v_{n-1}\left(t_{i}\right)\right) .
$$

Let $n \rightarrow \infty$, then by the Lebesgue-dominated convergence theorem, for $t \in J_{k}$, we have

$$
\underline{u}(t)=S(t) u_{0}+\int_{0}^{t} S(t-s)(f(s, \underline{u}(s), G \underline{u}(s))+C \underline{u}(s)) d s+\sum_{0<t_{i}<t} S\left(t-t_{i}\right) I_{i}\left(\underline{u}\left(t_{i}\right)\right),
$$

and $\underline{u} \in C\left(J_{k}, E\right)$. Therefore, for any $t \in J$, we have

$$
\underline{u}(t)=S(t) u_{0}+\int_{0}^{t} S(t-s)(f(s, \underline{u}(s), G \underline{u}(s))+C \underline{u}(s)) d s+\sum_{0<t_{i}<t} S\left(t-t_{i}\right) I_{i}\left(\underline{u}\left(t_{i}\right)\right) .
$$

Namely, $\underline{u} \in \operatorname{PC}(J, E)$, and $\underline{u}=Q \underline{u}$. Similarly, we can prove that there exists $\bar{u} \in \operatorname{PC}(J, E)$ such that $\bar{u}=Q \bar{u}$. By the monotonicity of operator $Q$, it is easy to prove that $\underline{u}$ and $\bar{u}$ are the minimal and maximal fixed points of $Q$ in $\left[v_{0}, w_{0}\right]$, and they are the minimal and maximal mild solutions of $\operatorname{IVP}(1.1)$ in $\left[v_{0}, w_{0}\right]$, respectively. 
Now we discuss the uniqueness of mild solutions for $\operatorname{IVP}(1.1)$ in $\left[v_{0}, w_{0}\right]$. If we replace assumption $\left(H_{3}\right)$ by the following assumption:

$\left(H_{4}\right)$ there exist positive constants $C_{1}$ and $C_{2}$ such that

$$
f\left(t, x_{2}, y_{2}\right)-f\left(t, x_{1}, y_{1}\right) \leq C_{1}\left(x_{2}-x_{1}\right)+C_{2}\left(y_{2}-y_{1}\right)
$$

for any $t \in J, v_{0}(t) \leq x_{1} \leq x_{2} \leq w_{0}(t), G v_{0}(t) \leq y_{1} \leq y_{2} \leq G w_{0}(t)$, and we have the following existence result.

Theorem 2.4. Let $E$ be an ordered Banach space, whose positive cone $P$ is normal, $A$ be a closed linear operator in $E,-A$ generate a positive $C_{0}$-semigroup $T(t)(t \geq 0), f \in C(J \times E \times E, E)$ and $I_{k} \in C(E, E), k=1,2, \ldots, m$. If IVP $(1.1)$ has a lower solution $v_{0}$ and an upper solution w with $v_{0} \leq w_{0}$, such that the assumptions $\left(H_{1}\right),\left(H_{2}\right)$, and $\left(H_{4}\right)$ are satisfied, then IVP $(1.1)$ has a unique mild solution between $v_{0}$ and $w_{0}$, which can be obtained by a monotone iterative procedure starting from $v_{0}$ or $w_{0}$.

Proof of Theorem 2.4. We firstly prove that $\left(H_{1}\right)$ and $\left(H_{4}\right)$ imply $\left(H_{3}\right)$. For $t \in J$, let $\left\{x_{n}\right\} \subset$ $\left[v_{0}(t), w_{0}(t)\right]$ and $\left\{y_{n}\right\} \subset\left[G v_{0}(t), G w_{0}(t)\right]$ be two increasing sequences. For $m, n \in \mathbb{N}$ with $m>n$, by $\left(H_{1}\right)$ and $\left(H_{4}\right)$, we have

$$
\theta \leq\left(f\left(t, x_{m}, y_{m}\right)-f\left(t, x_{n}, y_{n}\right)\right)+C\left(x_{m}-x_{n}\right) \leq\left(C_{1}+C\right)\left(x_{m}-x_{n}\right)+C_{2}\left(y_{m}-y_{n}\right) .
$$

By the normality of cone $P$, we have

$$
\left\|f\left(t, x_{m}, y_{m}\right)-f\left(t, x_{n}, y_{n}\right)\right\| \leq\left(C+N C+N C_{1}\right)\left\|x_{m}-x_{n}\right\|+N C_{2}\left\|y_{m}-y_{n}\right\| .
$$

From this inequality and the definition of the measure of noncompactness, it follows that

$$
\alpha\left(\left\{f\left(t, x_{n}, y_{n}\right)\right\}\right) \leq\left(C+N C+N C_{1}\right) \alpha\left(x_{n}\right)+N C_{2} \alpha\left(y_{n}\right) \leq L\left(\alpha\left(\left\{x_{n}\right\}\right)+\alpha\left(\left\{y_{n}\right\}\right)\right),
$$

where $L=C+N C+N C_{1}+N C_{2}$. If $\left\{x_{n}\right\}$ and $\left\{y_{n}\right\}$ are two decreasing sequences, the above inequality is also valid. Hence $\left(H_{3}\right)$ holds.

Therefore, by Theorem 1.1, IVP(1.1) has minimal solution $\underline{u}$ and maximal solution $\bar{u}$ in $\left[v_{0}, w_{0}\right]$. By the proof of Theorem 1.1, (2.10)-(2.13) are valid. Going from $J_{1}$ to $J_{m+1}$ interval by interval we show that $\underline{u} \equiv \bar{u}$ on every $J_{k}$. 
For $t \in J_{1}$, by (2.10) and assumption $\left(H_{4}\right)$, we have

$$
\begin{aligned}
\theta & \leq \bar{u}(t)-\underline{u}(t)=Q \bar{u}(t)-Q \underline{u}(t) \\
& =\int_{0}^{t} S(t-s)(f(s, \bar{u}(s), G \bar{u}(s))-f(s, \underline{u}(s), G \underline{u}(s))+C(\bar{u}(s)-\underline{u}(s))) d s \\
& \leq \int_{0}^{t} S(t-s)\left(\left(C+C_{1}\right)(\bar{u}(s)-\underline{u}(s))+C_{2}(G \bar{u}(s)-G \underline{u}(s))\right) d s \\
& \leq M\left(C+C_{1}+a C_{2} K_{0}\right) \int_{0}^{t}(\bar{u}(s)-\underline{u}(s)) d s .
\end{aligned}
$$

From this inequality and the normality of cone $P$, it follows that

$$
\|\bar{u}(t)-\underline{u}(t)\| \leq N M\left(C+C_{1}+a C_{2} K_{0}\right) \int_{0}^{t}\|\bar{u}(s)-\underline{u}(s)\| d s .
$$

By the Gronwall-Bellman inequality, we obtain that $\bar{u}(t) \equiv \underline{u}(t)$ on $J_{1}$.

For $t \in J_{2}$, since $I_{1}\left(\bar{u}\left(t_{1}\right)\right)=I_{1}\left(\underline{u}\left(t_{1}\right)\right)$, using (2.10) and the same argument as above for $t \in J_{1}$, we can prove that

$$
\begin{aligned}
\|\bar{u}(t)-\underline{u}(t)\| & \leq N M\left(C+C_{1}+a C_{2} K_{0}\right) \int_{0}^{t}\|\bar{u}(s)-\underline{u}(s)\| d s \\
& =N M\left(C+C_{1}+a C_{2} K_{0}\right) \int_{t_{1}}^{t}\|\bar{u}(s)-\underline{u}(s)\| d s .
\end{aligned}
$$

Again, by the Gronwall-Bellman inequality, we obtain that $\bar{u}(t) \equiv \underline{u}(t)$ on $J_{2}$.

Continuing such a process interval by interval up to $J_{m+1}$, we see that $\bar{u}(t) \equiv \underline{u}(t)$ over the whole $J$. Hence, $u^{*}:=\bar{u}=\underline{u}$ is the unique mild solution of $\operatorname{IVP}(1.1)$ in $\left[v_{0}, w_{0}\right]$, which can be obtained by a monotone iterative procedure starting from $v_{0}$ or $w_{0}$.

\section{Acknowledgments}

The author is very grateful to the reviewers for their helpful comments and suggestions. The paper was supported by NNSF of China (10871160), the NSF of Gansu Province (0710RJZA103), and the Project of NWNU-KJCXGC-3-47.

\section{References}

[1] V. Lakshmikantham, D. D. BaĬnov, and P. S. Simeonov, Theory of Impulsive Differential Equations, vol. 6 of Series in Modern Applied Mathematics, World Scientific, Teaneck, NJ, USA, 1989.

[2] D. D. BaInnov and P. S. Simeonov, Systems with Impulse Effect. Stability, Theory and Applications, Ellis Horwood Series: Mathematics and Its Applications, Ellis Horwood, Chichester, UK, 1989.

[3] D. D. Bainov and P. S. Simeonov, Impulsive Differential Equations: Periodic Solutions and Applications, Longman, Harlow, UK, 1993. 
[4] D. Bainov and V. Covachev, Impulsive Differential Equations with a Small Parameter, vol. 24 of Series on Advances in Mathematics for Applied Sciences, World Scientific, River Edge, NJ, USA, 1994.

[5] A. M. Samoilenko and N. A. Perestyuk, Differential Equations with Impulse Effect, Viska Skoda, Kiev, Russia, 1987.

[6] J. H. Liu, "Nonlinear impulsive evolution equations," Dynamics of Continuous, Discrete and Impulsive Systems Series B, vol. 6, no. 1, pp. 77-85, 1999.

[7] A. Anguraj and M. M. Arjunan, "Existence and uniqueness of mild and classical solutions of impulsive evolution equations," Electronic Journal of Differential Equations, vol. 2005, no. 111, pp. 1$8,2005$.

[8] T. Cardinali and P. Rubbioni, "Mild solutions for impulsive semilinear evolution differential inclusions," Journal of Applied Functional Analysis, vol. 1, no. 3, pp. 303-325, 2006.

[9] Y. H. Du, "Fixed points of increasing operators in ordered Banach spaces and applications," Applicable Analysis, vol. 38, no. 1-2, pp. 1-20, 1990.

[10] A. Pazy, Semigroups of Linear Operators and Applications to Partial Differential Equations, vol. 44 of Applied Mathematical Sciences, Springer, New York, NY, USA, 1983.

[11] K. Deimling, Nonlinear Functional Analysis, Springer, Berlin, Germany, 1985.

[12] H.-P. Heinz, "On the behaviour of measures of noncompactness with respect to differentiation and integration of vector-valued functions," Nonlinear Analysis: Theory, Methods E Applications, vol. 7, no. 12, pp. 1351-1371, 1983. 


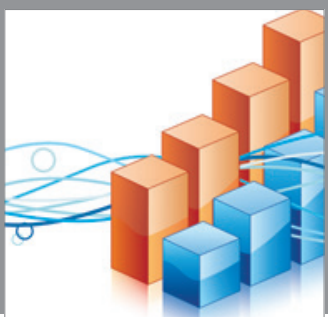

Advances in

Operations Research

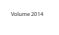

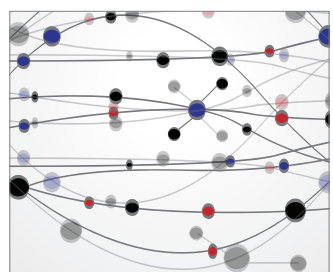

\section{The Scientific} World Journal
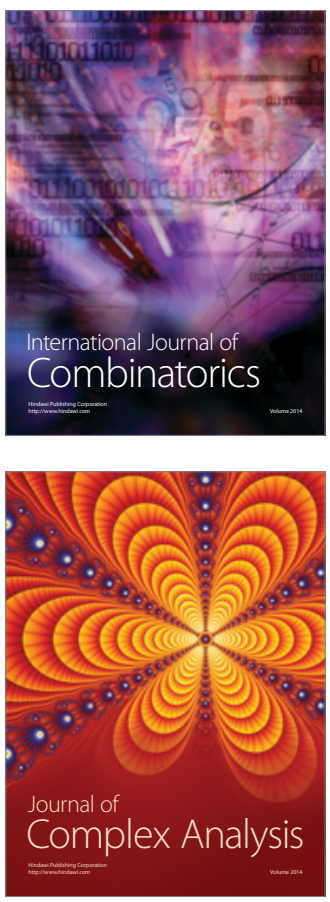

International Journal of

Mathematics and

Mathematical

Sciences
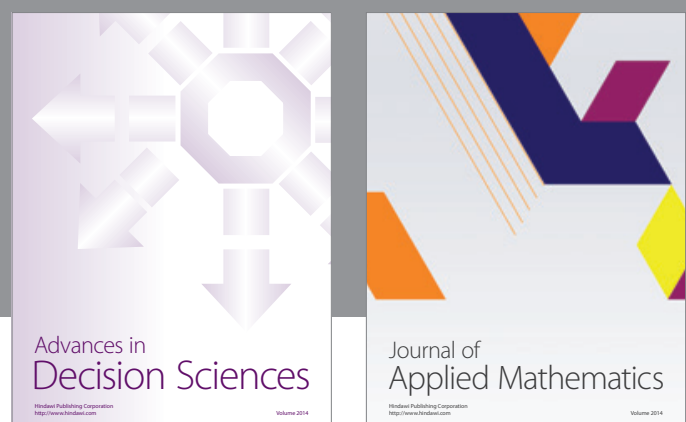

Journal of

Applied Mathematics
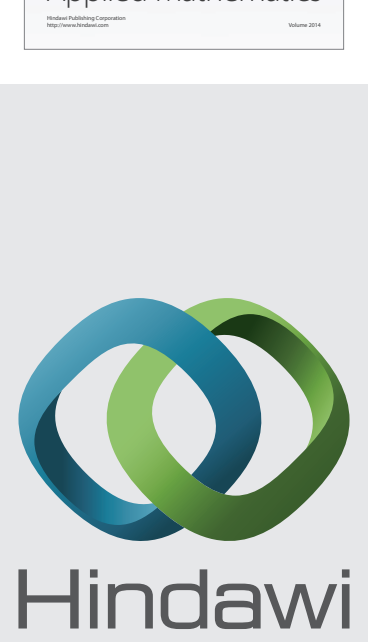

Submit your manuscripts at http://www.hindawi.com
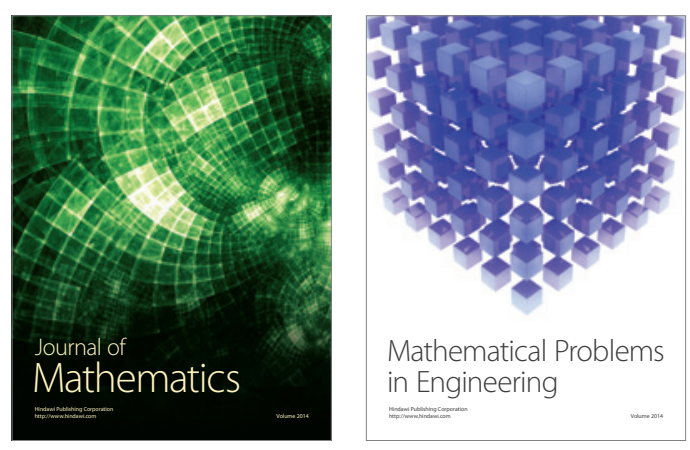

Mathematical Problems in Engineering
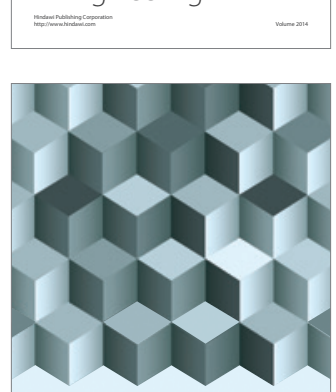

Journal of

Function Spaces
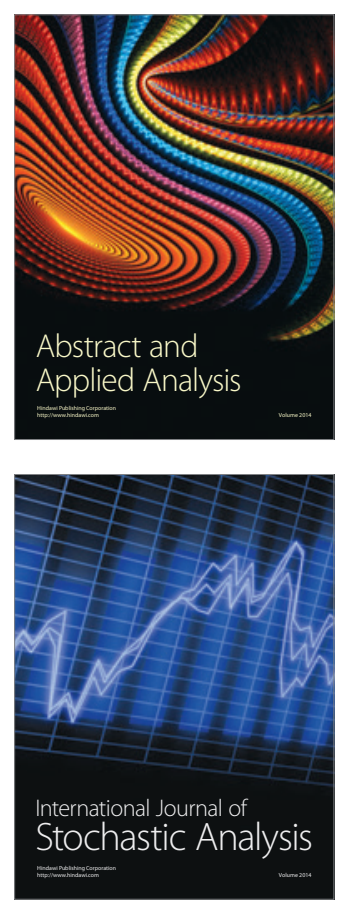

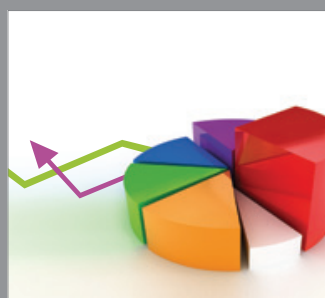

ournal of

Probability and Statistics

Promensencen
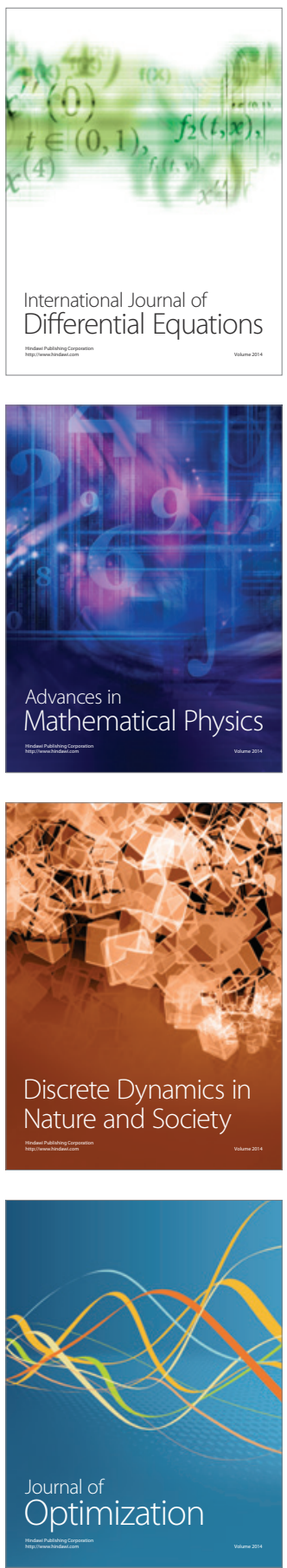